"This accepted author manuscript is copyrighted and published by Elsevier. It is posted here by agreement between Elsevier and MTA. The definitive version of the text was subsequently published in Molecular Immunology. 2010 Apr;47(7-8):1476-83. doi: 10.1016/j.molimm.2010.02.002.. Available under license CC-BY-NC-ND."

\title{
Anti-factor B autoantibody in dense deposit disease
}

Stefanie Strobel ${ }^{1}$, Miriam Zimmering ${ }^{2, \#}$, Krisztián Papp ${ }^{3}$, József Prechl ${ }^{3}$, Mihály Józsi ${ }^{1, *}$

${ }^{1}$ Junior Research Group Cellular Immunobiology, Leibniz Institute for Natural Product Research and Infection Biology - Hans Knöll Institute, Jena, Germany;

${ }^{2}$ Department of Pediatrics, Division of Nephrology, Charité Berlin, Berlin, Germany;

${ }^{3}$ Immunology Research Group, Hungarian Academy of Sciences, Budapest, Hungary

\# present address: Kinder Reha-Zentrum Usedom, Kölpinsee, Germany

*Corresponding author: Mihály Józsi, Junior Research Group Cellular Immunobiology, Leibniz Institute for Natural Product Research and Infection Biology - Hans Knöll Institute, Beutenbergstr. 11a, D-07745 Jena, Germany; Phone: +49 36415321720 ; Fax: +49 3641 5320815; E-mail: mihaly.jozsi@hki-jena.de.

Abbreviations: C3NeF, C3 nephritic factor; CFB, complement factor B; DDD, dense deposit disease; MPGN, membranoproliferative glomerulonephritis 


\begin{abstract}
Dense deposit disease (DDD), also known as membranoproliferative glomerulonephritis type II, is a rare kidney disorder that is associated with dysregulation of the alternative pathway of complement. Autoantibodies against the C3bBb convertase termed C3 nephritic factor are common in DDD patients. Here we report an autoantibody that binds to complement factor B in a DDD patient who was negative for C3 nephritic factor. This anti-factor B autoantibody recognized an epitope within the $\mathrm{Bb}$ fragment and was able to bind to the $\mathrm{C} 3 \mathrm{bBb}$ convertase. Upon binding, the anti-factor B autoantibody stabilized the convertase against both intrinsic and factor H-mediated extrinsic decay, and thus enhanced C3 consumption. Functional analyses demonstrated that, in contrast to C3 nephritic factor, the anti-factor B autoantibody inhibited complement-mediated lysis in vitro due to inhibition of the C5 convertase and the terminal complement pathway. Analysis of $\mathrm{C} 5 \mathrm{a}$ plasma levels indicated that not all $\mathrm{C} 5$ convertases are inhibited by the autoantibodies in the patient in vivo. Antigen array experiments confirmed the presence of anti-factor B autoantibodies and also revealed complement activating anti-C1q antibodies in the patient's plasma. In summary, the present report describes a new autoantibody in DDD that binds to factor B and to the alternative pathway C3 convertase and alters the kinetics of complement activation and regulation.
\end{abstract}

Keywords: Dense deposit disease; Membranoproliferative glomerulonephritis; Complement; Autoantibody; Factor B; C3 nephritic factor 


\section{Introduction}

Dense deposit disease (DDD), also termed membranoproliferative glomerulonephritis type II (MPGN II), is a rare renal disorder that may present with hematuria, proteinuria, acute nephritic syndrome or nephrotic syndrome, and leads to end-stage renal disease in most of the affected individuals (Appel et al., 2005). Kidney biopsies show capillary wall thickening, mesangial cell proliferation and expansion of the mesangial matrix. Immunofluorescence microscopy shows staining for complement C3 usually in the absence of immunoglobulins (Smith et al., 2007). The diagnostic hallmark of DDD is the deposition of electron-dense material in the glomerular basement membrane. DDD is often accompanied by hypocomplementemia, which is indicative of the role of the alternative complement pathway in the pathogenesis of DDD (Benz and Amann, 2009).

The complement system, which consists of $\sim 35$ proteins, is a rapidly activated protein cascade that plays an important role in host defense against infections and in inflammatory processes (Walport 2001; Markiewski and Lambris, 2007). Complement can be activated via three major pathways, of which the alternative pathway shows a continuous low-level activation in plasma. Spontaneous hydrolysis of the C3 internal thioester bond results in C3( $\left.\mathrm{H}_{2} \mathrm{O}\right)$, which is able to bind factor B (Pangburn et al., 1981). After cleavage of factor B by factor $\mathrm{D}, \mathrm{C} 3\left(\mathrm{H}_{2} \mathrm{O}\right) \mathrm{Bb}$ forms the short-lived initial fluid-phase C3 convertase enzyme, which cleaves C3 into the anaphylatoxin C3a and the opsonin C3b that covalently binds to target surfaces and initiates the formation of the $\mathrm{C} 3 \mathrm{bBb}$ convertase. This leads to further $\mathrm{C} 3$ cleavage, C3b production and the generation of more $\mathrm{C} 3 \mathrm{bBb}$. In order to limit convertase activity to target surfaces (e.g. microbes), the convertase is regulated in the host by serum proteins such as factor $\mathrm{H}$ and factor $\mathrm{I}$, and also by membrane-bound regulators. Mutations in complement genes have been linked to MPGN (Licht and Fremeaux-Bacchi, 2009). In particular, quantitative or functional factor $\mathrm{H}$ deficiency, due to mutations in the $C F H$ gene or 
autoantibody against the regulatory domains of factor H, lead to MPGN (Ault et al.,1997; Dragon-Durey et al., 2004; Licht et al., 2006; Pickering et al., 2002; Meri et al., 1992).

Two forms of autoimmune DDD are known and in both cases autoantibodies affect complement regulation. An anti-factor $\mathrm{H}$ miniantibody consisting of lambda light-chains, which inhibited factor $\mathrm{H}$ complement regulatory activity, was identified in a DDD patient (Meri et al., 1992; Jokiranta et al., 1999). C3 nephritic factor (C3NeF), an antibody against the C3bBb convertase (Spitzer et al., 1969), is found in 50-80\% of DDD patients (Schwertz et al., 2001; Appel et al., 2005). C3NeF binds to a neoepitope on the newly assembled C3bBb, increases the half-life of the convertase against both intrinsic and extrinsic, factor H-mediated, decay and thus enhances C3 consumption (Daha et al., 1976). However, C3NeF is also found in patients with partial lipodystrophy and even in healthy individuals; therefore, its pathogenic role is unclear (Appel et al., 2005; Mathieson et al., 1993). In addition, C3NeF is usually identified by hemolysis assays (Rother, 1982) or by C3 breakdown products (Davies and Norsworthy, 2000), but not by measuring autoantibody binding to the C3 convertase directly, which brings uncertainty into the interpretation of literature data.

In the present study, we identify and characterize a novel autoantibody in DDD against factor $\mathrm{B}$ in a C3NeF-negative patient. The nature and functional effects of this anti-factor B autoantibody are in part distinct from that of $\mathrm{C} 3 \mathrm{NeF}$, because it binds to native factor $\mathrm{B}$, binds to and stabilizes the C3 convertase, but inhibits the terminal pathway. 


\section{Methods}

These studies have been approved by the Research Ethics Committee of the Medical Faculty of Friedrich Schiller University, and were performed in accordance with the Declaration of Helsinki.

\subsection{Microtiter plate assays}

Autoantibodies were analyzed by ELISA as described (Józsi et al., 2007), using factor B, factor H, factor I, C3 and C3b (Merck Biosciences, Schwalbach, Germany) immobilized at 5 $\mu \mathrm{g} / \mathrm{ml}$. IgG subtypes were identified using anti-IgG1-IgG4 mAbs or mAbs against lambda and kappa light chains (Sigma-Aldrich, Taufkirchen, Germany). For inhibition experiments, the patient's plasma was incubated with factor B or C3 before adding to factor B-coated wells or the immobilized factor B was preincubated with factor B- and factor H-specific goat antibody (Merck Biosciences) and anti-Ba or anti-Bb mAbs (Quidel; TECOMedical GmbH, Bünde, Germany) before the patient's plasma was added to the wells. IgG was isolated using Protein G columns (GE Healthcare, Freiburg, Germany). To remove IgG, plasma samples were incubated with Protein G-agarose beads (Sigma-Aldrich) for $30 \mathrm{~min}$ at $20^{\circ} \mathrm{C}$. Factor B level in plasma was measured by ELISA using anti-Bb mAb as catch antibody and polyclonal antifactor B as detection antibody. C5a and soluble C5b-9 (sC5b-9) levels in plasma samples were measured by ELISA (Quidel).

\subsection{Solid-phase C3 convertase assays}

The C3bBb C3 convertase was generated on MaxiSorp microtiter plates (Nunc, Wiesbaden, Germany) as described (Hourcade et al., 2002), in DPBS containing 0.1\% Tween-20, 4\% BSA and $2 \mathrm{mM} \mathrm{NiCl}_{2}$. C3b was immobilized at $5 \mu \mathrm{g} / \mathrm{ml}$ at $4^{\circ} \mathrm{C}$ overnight. After washing, 1 $\mu \mathrm{g} / \mathrm{ml}$ factor B was added together with $250 \mathrm{ng} / \mathrm{ml}$ factor D and $500 \mathrm{ng} / \mathrm{ml}$ properdin (Merck Biosciences) for $2 \mathrm{hr}$ at $37^{\circ} \mathrm{C}$ in $50 \mu \mathrm{l}$. A goat anti-human factor B antibody was used to detect 
C3bBb. Binding of patient-derived IgG to C3bBb was measured by HRP-conjugated antihuman IgG (Sigma-Aldrich). For testing the activity of the convertase, $10 \mu \mathrm{g} / \mathrm{ml} \mathrm{C3}$ was added for $60 \mathrm{~min}$ at $37^{\circ} \mathrm{C}$, and generated C3a was measured by ELISA (Quidel). Convertase decay in the absence or presence of $1 \mu \mathrm{g} / \mathrm{ml}$ factor $\mathrm{H}$ and $500 \mu \mathrm{g} / \mathrm{ml}$ IgG preparations was measured by detecting the remaining intact convertase using anti-factor B.

\subsection{Hemolysis assays}

$5 \times 10^{6}$ rabbit erythrocytes (BioTrend Chemikalien, Cologne, Germany) were incubated with $10 \%$ normal human plasma, in a final volume of $100 \mu \mathrm{l}$, diluted in HEPES-buffer (20 mM HEPES, 7 mM $\mathrm{MgCl}_{2}$, 10 mM EGTA, 144 mM NaCl, 1\% BSA, pH 7.4). Purified IgG was added in DPBS. After 30 min incubation at $37^{\circ} \mathrm{C}$, absorbance of the supernatants was measured at $414 \mathrm{~nm}$. C3a and C5a levels in the supernatants were measured by ELISA (Quidel).

\subsection{Generation and analysis of antigen array data}

Antigens were spotted onto nitrocellulose-covered FAST-slides (Whatman, Kent, UK) using BioOdyssey Calligrapher miniarrayer (BioRad, CA) then stored at $4^{\circ} \mathrm{C}$ in sealed bags. Dried arrays were rinsed in PBS for 15 min before use then incubated with $20 \%$ serum at $37^{\circ} \mathrm{C}$ for 1 hr providing suitable conditions for complement activation. Sera were diluted in veronal buffer supplemented with 5\% BSA, 0.05\% Tween 20, $\mathrm{Ca}^{2+}$ and $\mathrm{Mg}^{2+}$. Slides were washed with PBS containing $0.05 \%$ Tween 20 , and first incubated with goat anti-human IgG F(ab') 2- $^{-}$ biotin then, following washing, with a mixture of Alexa647-conjugated goat F(ab’) $)_{2}$ of antihuman C3 (MP Biomedicals), FITC-conjugated rabbit anti-human C1q (Dako), and PECy5.5-conjugated streptavidin (eBioscience). Labelling with antibodies was carried out at room temperature for $30 \mathrm{~min}$ in PBS containing 5\% BSA and 0.05\% Tween 20. After washing, arrays were dried and scanned on Typhoon Trio+ imager (Amersham Bioscience, 
München, Germany). Images were analyzed with GenePix Pro 6.0 (Molecular Devices, Corp.)

software. Intensity of fluorescence ( $\mathrm{FI}=\mathrm{FI}_{\text {median (pixels of spot) }}-\mathrm{FI}$ median (pixels of local background) $)$ was calculated for each spot; the mean of the replicates were used for further analysis. For interassay comparisons, C1q, C3 and IgG signals were normalized to printed C1q, Protein G, and human IgG spots, respectively.

\subsection{Analysis of anti-C1q autoantibodies}

$\mathrm{C} 1 \mathrm{q}$ in the IgG preparations was detected by measuring anti-C1q antibody (Merck Biosciences) binding to the immobilized IgG fractions $(20 \mu \mathrm{g} / \mathrm{ml})$. IgG binding from plasma samples (1:50) to immobilized C1q ( $2 \mu \mathrm{g} / \mathrm{ml})$ was measured by ELISA as described (Siegert et al., 1990). Samples were diluted in buffer containing $1 \mathrm{M} \mathrm{NaCl}$ to exclude interaction of C1q globular heads with IgG Fc parts. 


\section{Results}

\subsection{Patient description}

The patient, an 11-year-old girl, was diagnosed with DDD at the age of 6.3 years, four years after a postinfectious period of macrohematuria and proteinuria. She was treated with plasma exchanges and fresh frozen plasma, but renal function impaired and dialysis was necessary. Nevertheless, proteinuria and low C3 levels were further observed. Complement analysis showed reduced C3 level $(0.21 \mathrm{mg} / \mathrm{ml}$; normal range: 0.78-2.1 mg/ml) and increased C3d (726 mU/l; normal: <40 mU/l) amount in plasma. APH50 was 89\% whereas the CH50 was 32\%. The patient was tested negative for C3NeF by a hemolytic assay (Rother, 1982). Western blot analyses of plasma samples showed the presence of the complement activation fragments C3d and Ba. Factor B levels were $119 \mu \mathrm{g} / \mathrm{ml}$ and $66 \mu \mathrm{g} / \mathrm{ml}$ in two different samples (181 $\mu \mathrm{g} / \mathrm{ml}$ in pooled normal human plasma; normal range: $75-280 \mu \mathrm{g} / \mathrm{ml}$ ). There was no known family history of the disease. Genetic analysis of complement genes was not performed.

\subsection{Identification and characterization of an anti-factor B autoantibody}

Because of signs of complement dysregulation, we have analyzed if autoantibodies distinct from $\mathrm{C} 3 \mathrm{NeF}$ were present in the patient's plasma. Factor B, C3, C3b, factor I and factor $\mathrm{H}$ were immobilized on microtiter plate wells and the presence of IgG autoantibodies was tested as described before (Józsi et al., 2007). Anti-factor B IgG was detected only in the patient’s plasma but not in four samples from DDD patients with $\mathrm{C} 3 \mathrm{NeF}$ and in samples from 10 healthy controls (Fig. 1a, and data not shown). C3NeF binds to a neoepitope on the C3 convertase but not to its individual components (Daha and Van Es, 1981). In agreement with this, we found no IgG binding from four analyzed C3NeF-positive plasma samples to factor 
B, C3 or C3b. Thus, the autoantibody of this patient specifically interacts with factor B and is distinct from C3NeF.

The autoantibody showed dose-dependent binding to immobilized factor B from plasma (Fig. 1b), and the removal of the plasma IgG fraction abrogated factor B binding (Fig. 1c). IgG isolated from the patient's plasma bound to factor B in a dose-dependent manner (Fig. 1d). In addition, factor B:IgG complexes in plasma were detected using an anti-factor B antibody to catch the complexes (Fig. 1e). IgG-subtype analysis revealed IgG3 anti-factor B antibodies with kappa light chains (data not shown).

To confirm the specificity of the autoantibody, two types of blocking experiments were performed. The addition of purified factor B, but not of C3, as competitor soluble antigen to the patient's plasma inhibited autoantibody binding (Fig. 2a). Likewise, polyclonal antibodies against factor B inhibited autoantibody reactivity with factor B, but a polyclonal anti-factor $\mathrm{H}$ antibody, used as a control, failed to block binding (Fig. 2b).

\subsection{Localization of the autoantibody binding site on factor $B$}

In order to determine the binding site of the autoantibody, immobilized factor B was preincubated with monoclonal antibodies (mAbs) against the factor $\mathrm{B}$ fragments $\mathrm{Ba}$ and $\mathrm{Bb}$ before adding plasma samples to the wells. The anti-Bb mAb inhibited autoantibody binding to factor B in a dose-dependent manner, whereas the anti-Ba mAb had no effect, indicating that the larger $\mathrm{Bb}$ fragment contains the autoantibody binding epitope (Fig. 3).

\subsection{The anti-factor B autoantibody binds to and stabilizes the C3 convertase}

$\mathrm{Bb}$ is the enzymatically active part of the $\mathrm{C} 3 \mathrm{bBb}$ convertase, therefore we have investigated whether the anti-factor B autoantibody binds to this enzyme. The solid-phase C3 convertase was built up by adding factor B in the presence of factor D and properdin to C3b-coated wells (Fig. 4a). To confirm the formation of an active convertase, C3 was added to the wells and C3 
cleavage was analyzed by measuring the generated C3a fragment by ELISA (Fig. 4a, right panel). IgG isolated from the patient's plasma that contained the anti-factor B antibodies bound to the C3-convertase in a dose-dependent manner, similar to IgG derived from a C3NeF-positive MPGN patient. By contrast, IgG purified from healthy donors showed no binding (Fig. 4b). The anti-factor B autoantibody did not influence the assembly of the convertase (data not shown).

In functional assays, both the intrinsic and the factor $\mathrm{H}$-mediated extrinsic decay of the C3bBb convertase were analyzed. The surface-bound C3 convertase was incubated with IgG isolated from the patient and from controls in the presence or absence of factor $\mathrm{H}$, and the decay was followed for $60 \mathrm{~min}$ by measuring bound $\mathrm{Bb}$. The anti-factor B autoantibody stabilized the convertase against both spontaneous (Fig. 4c) and factor H-mediated decay (Fig. 4d), in a manner similar to C3NeF. IgG isolated from healthy individuals had no influence on convertase decay.

\subsection{The anti-factor B antibody affects the C5 convertase and the terminal pathway}

Stabilization of the $\mathrm{C} 3$ convertase by $\mathrm{C} 3 \mathrm{NeF}$ results in enhanced $\mathrm{C} 3$ turnover and, consequently, in an increased complement activation. To analyze the effect of the anti-factor B autoantibody, alternative pathway mediated hemolysis was measured using rabbit red blood cells (RRBC). RRBC are lysed in normal human plasma because they lack regulators to protect them from human complement-mediated lysis. The anti-factor B autoantibody, when added to human plasma, caused a strong inhibition of RRBC lysis in a dose-dependent manner, in contrast to the lysis-enhancing effect of C3NeF (Fig. 5a and b). Similar inhibitory effect was seen with polyclonal anti-factor B and anti-C3 antibodies.

The levels of C3a and C5a were analyzed in these samples as a measure of C3- and C5 convertase activities. Whereas the C3a levels were increased due to the stabilized convertase in proportion to the amount of added autoantibody (Fig. 5c), C5a generation was dose- 
dependently inhibited, indicating an inhibitory effect of the anti-factor B autoantibody on the C3bBbC3b alternative pathway C5 convertase (Fig. 5d). Thus, although both autoantibodies bind to the C3 convertase and enhance C3 consumption, the anti-factor B autoantibody inhibits alternative pathway mediated cell lysis, whereas C3NeF enhances the hemolysis.

To further analyze the effect of the autoantibody on the terminal pathway in the patient, C5a and soluble C5b-9 (sC5b-9) levels were determined in plasma samples. C5a level was $16 \mathrm{ng} / \mathrm{ml}$ in the initial sample and it was strongly increased $(231 \mathrm{ng} / \mathrm{ml})$ in a sample collected two years later and containing similarly high amounts of anti-factor B autoantibodies. C5a amounts in normal plasma samples, determined in parallel, were below $10 \mathrm{ng} / \mathrm{ml}$. sC5b-9 levels were slightly higher $(0.9-2.2 \mu \mathrm{g} / \mathrm{ml})$ than in control plasma samples (ranging 0.1-1.6 $\mu \mathrm{g} / \mathrm{ml}$ ). These data indicated that, although patient-derived purified IgG can inhibit the terminal pathway in vitro, the autoantibodies in the plasma of the patient do not block all C5 convertases and the terminal pathway can still be activated.

\subsection{Antigen microarray analysis of autoantibodies}

We set out to further characterize the autoantibodies of the patient directly from plasma. In addition, because IgG3 antibodies are known as a complement activating IgG isotype, we wanted to examine the possibility that the anti-factor B autoantibody plays a pathogenic role in the patient by activating the classical pathway. A protein microarray was used to characterize the functional autoantibody profile of the patient (Papp et al., 2007). In this assay antigens are exposed to whole serum, thus this approach better mimics the in vivo situation for the formation of antigen-antibody complexes than do purified antibodies in ELISA. IgG binding and C3 deposition to a wide array of immobilized antigens from the patient's plasma were determined in parallel. The presence of anti-factor B autoantibodies was confirmed and no increased IgG signal was detected at the spots of C3, factor H, factor I and properdin (data not shown). Although there was slightly higher C3 deposition on factor B spots from the 
patient's plasma compared to normal control, much higher C3 deposition and IgG binding were detected on C1q, implying either the presence of complement activating anti-C1q antibodies or C3-containing circulating immune complexes (Fig. 6a). These analyses indicated no pathologically significant complement activation by the amounts and type of anti-factor B autoantibodies present in the patient's plasma.

Next, the presence of C1q in the patient's IgG was analyzed. C1q was present in high amounts in the patient's IgG preparation as compared to IgG from healthy controls, suggesting the presence of immune complexes and/or anti-C1q autoantibodies in the patient (Fig. 6b). In addition, IgG binding to immobilized C1q was measured in $1 \mathrm{M} \mathrm{NaCl}$ to detect anti-C1q autoantibodies and to exclude C1q-IgG Fc interactions via the C1q globular heads (Siegert et al., 1990). This assay indicated that the patient's plasma contained autoantibodies against the collagen-like region of C1q (Fig. 6c). 


\section{Discussion}

In this report we describe a new anti-factor B autoantibody in DDD that binds to and stabilizes the C3 convertase and thus causes enhanced C3 consumption, but in contrast to C3NeF it interferes with terminal pathway activation.

The C3 convertase is central to the amplification of the alternative complement pathway by activating several C3 molecules through enzymatic cleavage. C3NeF, an autoantibody found in most MPGN patients, increases the half-life of the convertase by stabilizing it against decay, thus causing an increased C3 turnover. By changing the balance of complement activation and inhibition $\mathrm{C} 3 \mathrm{NeF}$ contributes to the disease, in line with the involvement of complement dysregulation in DDD (Appel et al., 2005). C3NeF, however, is also found in healthy persons and in other pathological conditions, and a clear causative relationship for C3NeF and DDD has not been demonstrated (Schwertz et al., 2001; Appel et al., 2005; Smith et al., 2007; Spitzer et al., 1990). The appearance of high-affinity C3NeF in the patients is possibly antigen-driven, due to neoepitopes exposed on activated complement components (Spitzer et al., 1992).

The anti-factor B autoantibody described here binds to native factor B as well as to the $\mathrm{Bb}$ fragment that is part of the alternative pathway convertase. Even though the patient was negative for an anti-convertase antibody, i.e. C3NeF, in the traditional hemolysis assay used to detect C3NeF, an antibody against factor B was identified by ELISA. This autoantibody, similar to $\mathrm{C} 3 \mathrm{NeF}$, bound to and stabilized a solid-phase $\mathrm{C} 3 \mathrm{bBb}$ convertase and its decay inhibitory effect translated into enhanced C3 consumption. However, in contrast to C3NeF, the anti-factor B autoantibody reduced C5 turnover in vitro due to a C5 convertase (C3bBbC3b) blocking effect (Figs. 4 and 5), probably by preventing the binding of the second C3b molecule. This difference is explained by the different binding sites of the two types of autoantibodies. The analyzed C3NeF IgGs failed to bind to factor B, C3 and C3b

(Fig. 1), consistent with C3NeF binding to a neoepitope on C3bBb (Daha and Van Es, 1981). 
C3NeF likely binds to a neoantigenic site on $\mathrm{Bb}$ but it is unable to bind to native factor $\mathrm{B}$ (Daha and Van Es, 1981). Thus, the two autoantibodies bind to different parts of the convertase.

Previously, two monoclonal antibodies raised against factor B were shown to mimic the effects of $\mathrm{C} 3 \mathrm{NeF}$, but a third monoclonal antibody rather facilitated the disassembly of the convertase (Daha et al., 1984). Similarly, the various autoantibodies against the C3 convertase or its components may differ in their exact binding sites and functional effects. A heterogeneity of $\mathrm{C} 3 \mathrm{NeF}$ is indeed observed and likely explains the differences in complement profiles between DDD patients (Appel et al., 2005; Davis et al., 1977; Daha et al., 1978; Mollnes et al., 1986).

Interpretation of reported $\mathrm{C} 3 \mathrm{NeF}$ cases is difficult, due to the apparent heterogeneous nature of these autoantibodies, the scarcity of detailed functional studies, and the various methods used for C3NeF measurement. A direct detection of autoantibodies is often not performed, and the presence of anti-convertase autoantibodies is deduced from hemolysis assays. However, an antibody against the C3 convertase that at the same time inhibits the C5 convertase would be undetected with this method, as shown here (Figs. 1 and 5). In addition, the C3 convertase could be stabilized by other means. For instance, gain-of-function mutations in factor B and C3 also lead to more stable convertases and higher C3 turnover (Goicoechea de Jorge et al., 2007; Roumenina et al., 2009; Frémeaux-Bacchi et al., 2008). Our results emphasize the importance of measuring antibodies directly. ELISA assays should complement the commonly used hemolysis assay to identify convertase-specific and additional anti-complement antibodies. Such solid-phase assay is also suitable to detect the antibody-convertase complexes, thus can be used to identify C3NeF (Seino et al., 1987).

Since in vitro experimental data demonstrated a C3 convertase stabilizing and C5 convertase inhibitory effect of the purified autoantibodies, we have also analyzed plasma samples for signs of terminal pathway activation. These data showed that the terminal 
pathway can still be activated in the patient's plasma, thus not all C5 convertases are inhibited in vivo by the amounts of anti-factor B autoantibodies present. In addition, because this autoantibody affects only the alternative pathway convertases and cannot bind to classical pathway convertases, the terminal pathway remains functional.

Since factor B:IgG complexes were detected in the patient, and the autoantibodies were of IgG3 isotype, we investigated the possibility of immune complex-mediated classical pathway activation by analyzing the complement activatory capacity of the autoantibodies on antigen microarray (Papp et al., 2007). The observed, somewhat elevated C3 deposition on factor B spots from the patient’s plasma are unlikely to be of major pathological significance, because neither factor B nor IgG are detected in glomerular deposits in DDD (Sethi et al., 2009). Although the amount of C1q was increased in the IgG fraction of the patient, this represented anti-C1q autoantibodies (Fig. 6). In addition, the microarray experiments revealed enhanced antibody and C1q binding to nuclear antigens, such as DNA and RNA, indicating systemic autoimmune processes (data not shown). Autoantibodies against complement components play a critical role in the pathophysiology of several diseases including systemic lupus erythematosus, atypical hemolytic uremic syndrome and MPGN (Trouw et al., 2001, 2003). Anti-C1q autoantibodies in MPGN have been reported before (Strife et al., 1989, 1990), and such antibodies are usually associated with activation of the classical pathway (Frémeaux-Bacchi et al., 1996), which explains the low CH50 value measured in the patient.

Our data demonstrate that antigen microarrays in combination with complement activity testing are a helpful tool to detect various autoantibodies in parallel and to interpret their role in autoimmune diseases. Whereas MPGN patients are commonly screened for C3NeF, identifying additional antibodies is equally important. In an analogous manner to $\mathrm{C} 3 \mathrm{NeF}$, antibodies that bind to the classical pathway convertases, termed C4NeF, may also affect complement regulation in the patients (Halbwachs et al., 1980). The presence of such additional antibodies may in part explain the differences between patients and the lack of the 
clear correlation between the presence and amounts of $\mathrm{C} 3 \mathrm{NeF}$ and the complement status and pathology in DDD patients.

Due to its stabilizing effect, the anti-factor B autoantibody causes increased C3 turnover that contributes to the hypocomplementemia and the C3 deposition in the glomeruli that is commonly observed in DDD. Analyses of glomerular deposits in DDD showed the presence of C3 degradation fragments, C5 and other terminal pathway components (West et al., 2001; Sethi et al., 2009), indicating a role for both the alternative and the terminal complement pathway. Animal studies comparing factor H-deficient mice and mice deficient in both factor $\mathrm{H}$ and $\mathrm{C} 5$ demonstrated that glomerulonephritis develops in the absence of C5 and terminal pathway activation, although the latter contribute to glomerular inflammation and a more severe pathology (Pickering et al., 2006). Factor B-autoantibody complexes are formed on solid-phase C3 convertases but are also present in fluid phase (Fig. 1e). Factor B and IgG, however, are not detected in glomerular deposits in DDD (Sethi et al., 2009). This suggests that factor B-autoantibody complexes, which could cause some degree of C3 activation (Fig. 6a), unlikely to be of pathological significance by direct complement activation, and that the autoantibodies affect C3 convertase activity outside the glomeruli. This is consistent with the established role of impaired fluid phase alternative pathway regulation in the pathogenesis of DDD. Thus, the anti-factor B autoantibody likely has a role in DDD by altering C3 convertase regulation in plasma, while at the same time not leading to C5 consumption. This could be a reason for the normal APH50 level measured in the patient.

In the present study, it was not possible to systematically analyze samples and determine when the autoantibodies appeared in the patient, thus whether they were in a direct causative relationship with the disease manifestation. It is more likely that the anti-factor B antibody was only one of the factors that contributed to the disease by enhancing C3 turnover.

In summary, we identify a new autoantibody in DDD that acts on the alternative pathway C3 convertase and alters the kinetics of complement activation and regulation. The 
major differences between the anti-factor B antibody and C3NeF are that (i) the anti-factor B antibody is not detected by the hemolysis assay used to identify convertase-specific antibodies, (ii) the anti-factor $\mathrm{B}$ antibody binds to native factor $\mathrm{B}$ as well as to the $\mathrm{C} 3$ convertase, and (iii) the anti-factor B antibody interferes with the C5 convertase. These results provide rationale to design studies that address the presence and role of anti-factor $\mathrm{B}$ autoantibodies in DDD patients and establish their diagnostic and prognostic value. The development of new diagnostic tools and a better understanding of the mechanisms of complement dysregulation in the patients may lead to specific and effective treatments in the future. 


\section{Acknowledgments}

This work was supported in part by a grant from the Deutsche Forschungsgemeinschaft (JO 844/1-1). Antigen microarray research was supported by the Hungarian Academy of Sciences and Péter Pázmány Programme CellKom RET-06. We thank Peter F. Zipfel (Hans Knöll Institute, Jena) for providing plasma samples and for access to equipments. Part of this work was presented at the 12th European meeting on Complement in Human Disease, Visegrád, Hungary (Mol. Immunol. 46:2840-2841., abstract).

Author contributions: S.S. designed and performed research, analyzed data and wrote the paper; M.Z. was in charge of the patient, provided material and clinical data; K.P. and J.P. performed on-chip complement assays and data analysis; M.J. initiated the study, designed research, analyzed data and wrote the paper. 


\section{References}

Appel, G.B., Cook, H.T., Hageman, G., Jennette, J.C., Kashgarian, M., Kirschfink, M., Lambris, J.D., Lanning, L., Lutz, H.U., Meri, S., Rose, N.R., Salant, D.J., Sethi, S., Smith, R.J., Smoyer, W., Tully, H.F., Tully, S.P., Walker, P., Welsh, M., Würzner, R., Zipfel, P.F., 2005. Membranoproliferative glomerulonephritis type II (dense deposit disease): an update. J. Am. Soc. Nephrol. 16, 1392-1403.

Ault, B.H., Schmidt, B.Z., Fowler, N.L., Kashtan, C.E., Ahmed, A.E., Vogt, B.A., Colten, H.R., 1997. Human factor $\mathrm{H}$ deficiency. Mutations in framework cysteine residues and block in H protein secretion and intracellular catabolism. J. Biol. Chem. 272, 25168-25175.

Benz, K., Amann, K., 2009. Pathological aspects of membranoproliferative glomerulonephritis (MPGN) and haemolytic uraemic syndrome (HUS) / thrombocytic thrombopenic purpura (TTP). Thromb. Haemost. 101, 265-270.

Daha, M.R., Fearon, D.T., Austen, K.F., 1976. C3 nephritic factor (C3NeF): stabilization of fluid phase and cell-bound alternative pathway convertase. J. Immunol. 116, 1-7.

Daha, M.R., Austen, K.F., Fearon, D.T., 1978. Heterogeneity, polypeptide chain composition and antigenic reactivity of C3 nephritic factor. J. Immunol. 120, 1389-1394.

Daha, M.R., Van Es, L.A., 1981. Stabilization of homologous and heterologous cell-bound amplification convertases, C3bBb, by C3 nephritic factor. Immunology 43, 33-38. 
Daha, M.R., Deelder, A.M., Van Es, L.A., 1984. Stabilization of the amplification convertase of complement by monoclonal antibodies directed against human factor B. J. Immunol. 132, 2538-2542.

Davies, K.A., Norsworthy, P., 2000. Autoantibodies to complement components. Methods. Mol. Biol. 150, 173-192.

Davis, A.E., Ziegler, J.B., Gelfand, E.W., Rosen, F.S., Alper, C.A., 1977. Heterogeneity of nephritic factor and its identification as an immunoglobulin. Proc. Natl. Acad. Sci. U S A 74, 3980-3983.

Dragon-Durey, M-A., Frémeaux-Bacchi, V., Loirat, C., Blouin, J., Niaudet, P., Deschenes, G., Coppo, P., Herman Fridman, W., Weiss, L., 2004. Heterozygous and homozygous factor H deficiencies associated with hemolytic uremic syndrome or membranoproliferative glomerulonephritis: report and genetic analysis of 16 cases. J. Am. Soc. Nephrol. 15, 787-795.

Frémeaux-Bacchi, V., Weiss, L., Demouchy, C., Blouin, J., Kazatchkine, M.D., 1996. Autoantibodies to the collagen-like region of $\mathrm{C} 1 \mathrm{q}$ are strongly associated with classical pathway-mediated hypocomplementemia in systemic lupus erythematosus. Lupus 5, 216-220.

Frémeaux-Bacchi, V., Miller, E.C., Liszewski, M.K., Strain, L., Blouin, J., Brown, A.L., Moghal, N., Kaplan, B.S., Weiss, R.A., Lhotta, K., Kapur, G., Mattoo, T., Nivet, H., Wong, W., Gie, S., Hurault de Ligny, B., Fischbach, M., Gupta, R., Hauhart, R., Meunier, V., Loirat, C., Dragon-Durey, M.A., Fridman, W.H., Janssen, B.J., Goodship, T.H., Atkinson, J.P., 2008. Mutations in complement C3 predispose to development of atypical hemolytic uremic syndrome. Blood 112, 4948-4952. 
Goicoechea de Jorge, E., Harris, C.L., Esparza-Gordillo, J., Carreras, L., Arranz, E.A., Garrido, C.A., López-Trascasa, M., Sánchez-Corral, P., Morgan, B.P., Rodríguez de Córdoba, S., 2007. Gain-of-function mutations in complement factor B are associated with atypical hemolytic uremic syndrome. Proc. Natl. Acad. Sci. U S A 104, 240-245.

Halbwachs, L., Leveillé, M., Lesavre, P., Wattel, S., Leibowitch, J., 1980. Nephritic factor of the classical pathway of complement: immunoglobulin G autoantibody directed against the classical pathway C3 convetase enzyme. J. Clin. Invest. 65, 1249-1256.

Hourcade, D.E., Mitchell, L., Kuttner-Kondo, L.A., Atkinson, J.P., Medof, M.E., 2002. Decay-accelerating factor (DAF), complement receptor 1 (CR1), and factor $\mathrm{H}$ dissociate the complement AP C3 convertase (C3bBb) via sites on the type A domain of Bb. J. Biol. Chem. 277, 1107-1112.

Jokiranta, T.S., Solomon, A., Pangburn, M.K., Zipfel, P.F., Meri, S., 1999. Nephritogenic lambda light chain dimer: a unique human miniautoantibody against complement factor $\mathrm{H}$. J. Immunol. 163, 4590-4596.

Józsi, M., Strobel, S., Dahse, H-M., Liu, W.S., Hoyer, P.F., Oppermann, M., Skerka, C., Zipfel, P.F., 2007. Anti-factor $\mathrm{H}$ autoantibodies block C-terminal recognition function of factor H in hemolytic uremic syndrome. Blood 110, 1516-1518.

Licht, C., Heinen, S., Józsi, M., Löschmann, I., Saunders, R.E., Perkins, S.J., Waldherr, R., Skerka, C., Kirschfink, M., Hoppe, B., Zipfel, P.F., 2006. Deletion of Lys224 in regulatory 
domain 4 of Factor $\mathrm{H}$ reveals a novel pathomechanism for dense deposit disease (MPGN II). Kidney Int. 70, 42-50.

Licht, C., Fremeaux-Bacchi, V., 2009. Hereditary and acquired complement dysregulation in membranoproliferative glomerulonephritis. Thromb. Haemost. 101, 271-278.

Markiewski, M.M., Lambris, J.D., 2007. The role of complement in inflammatory diseases: from behind the scenes into the spotlight. Am. J. Pathol. 171, 715-727.

Mathieson, P.W., Würzner, R., Oliveria, D.B., Lachmann, P.J., Peters, D.K., 1993. Complement-mediated adipocyte lysis by nephritic factor sera. J. Exp. Med. 177, 1827-1831.

Meri, S., Koistinen, V., Miettinen, A., Törnroth, T., Seppälä, I.J., 1992. Activation of the alternative pathway of complement by monoclonal lambda light chains in membranoproliferative glomerulonephritis. J. Exp. Med. 175, 939-950.

Mollnes, T.E., Ng, Y.C., Peters, D.K., Lea, T., Tschopp, J., Harboe, M., 1986. Effect of nephritic factor on C3 and on the terminal pathway of complement in vivo and in vitro. Clin. Exp. Immunol. 65, 73-79.

Pangburn, M.K., Schreiber, R.D., Müller-Eberhard, H.J., 1981. Formation of the initial C3 convertase of the alternative complement pathway. Acquisition of C3b-like activities by spontaneous hydrolysis of the putative thioester in native C3. J. Exp. Med. 154, 856-867. 
Papp, K., Szekeres, Z., Terényi, N., Isaák, A., Erdei, A., Prechl, J., 2007. On-chip complement activation adds an extra dimension to antigen microarrays. Mol. Cell. Proteomics 6, 133-140.

Pickering, M.C., Cook, H.T., Warren, J., Bygrave, A.E., Moss, J., Walport, M.J., Botto, M., 2002. Uncontrolled C3 activation causes membranoproliferative glomerulonephritis in mice deficient in complement factor H. Nat. Genet. 31, 424-428.

Pickering, M.C., Warren, J., Rose, K.L., Carlucci, F., Wang, Y., Walport, M.J., Cook, H.T., Botto, M., 2006. Prevention of C5 activation ameliorates spontaneous and experimental glomerulonephritis in factor H-deficient mice. Proc. Natl. Acad. Sci. U S A. 103, 9649-9654.

Rother, U., 1982. A new screening test for C3 nephritis factor based on a stable cell bound convertase on sheep erythrocytes. J. Immunol. Methods 51, 101-107.

Roumenina, L.T., Jablonski, M., Hue, C., Blouin, J., Dimitrov, J.D., Dragon-Durey, M.A., Cayla, M., Fridman, W.H., Macher, M.A., Ribes, D., Moulonguet, L., Rostaing, L., Satchell, S.C., Mathieson, P.W., Sautes-Fridman, C., Loirat, C., Regnier, C.H., Halbwachs-Mecarelli, L., Fremeaux-Bacchi, V., 2009. Hyperfunctional C3 convertase leads to complement deposition on endothelial cells and contributes to atypical hemolytic uremic syndrome. Blood 114, 2837-2845.

Seino, J., Fukuda, K., Kinoshita, Y., Sudo, K., Horigome, I., Sato, H., Saito, T., Furuyama, T., Yoshinaga, K., 1987. Quantitation of C3 nephritic factor of alternative complement pathway by an enzyme-linked immunosorbent assay. J. Immunol. Methods 105, 119-125. 
Sethi, S., Gamez, J.D., Vrana, J.A., Theis, J.D., Bergen, H.R. 3rd, Zipfel, P.F., Dogan, A., Smith, R.J., 2009. Glomeruli of Dense Deposit Disease contain components of the alternative and terminal complement pathway. Kidney Int. 75, 952-960.

Siegert, C.E., Daha, M.R., van der Voort, E.A., Breedveld, F.C., 1990. IgG and IgA antibodies to the collagen-like region of $\mathrm{C} 1 \mathrm{q}$ in rheumatoid vasculitis. Arthritis Rheum. 33, 1646-1654.

Smith, R.J., Alexander, J., Barlow, P.N., Botto, M., Cassavant, T.L., Cook, H.T., de Córdoba, S.R., Hageman, G.S., Jokiranta, T.S., Kimberling, W.J., Lambris, J.D., Lanning, L.D., Levidiotis, V., Licht, C., Lutz, H.U., Meri, S., Pickering, M.C., Quigg, R.J., Rops, A.L., Salant, D.J., Sethi, S., Thurman, J.M., Tully, H.F., Tully, S.P., van der Vlag, J., Walker, P.D., Würzner, R., Zipfel, P.F.; Dense Deposit Disease Focus Group, 2007. New approaches to the treatment of dense deposit disease. J. Am. Soc. Nephrol. 18, 2447-2456.

Spitzer, R.E., Vallota, E.H., Forristal, J., Sudora, E., Stitzel, A., Davis, N.C., West, C.D., 1969. Serum C'3 lytic system in patients with glomerulonephritis. Science 164, 436-437.

Spitzer, R.E., Stitzel, A.E., Tsokos, G.C., 1990. Evidence that production of autoantibody to the alternative pathway C3 convertase is a normal physiologic event. J. Pediatr. 116, S103108.

Spitzer, R.E., Stitzel, A.E., Tsokos, G., 1992. On the origin of C3 nephritic factor (antibody to the alternative pathway C3 convertase): evidence for the Adam and Eve concept of autoantibody production. Clin. Immunol. Immunopathol. 64, 177-183. 
Strife, C.F., Leahy, A.E., West, C.D., 1989. Antibody to a cryptic, solid phase C1Q antigen in membranoproliferative nephritis. Kidney Int. 35, 836-842.

Strife, C.F., Prada, A.L., Clardy, C.W., Jackson, E., Forristal, J., 1990. Autoantibody to complement neoantigens in membranoproliferative glomerulonephritis. J. Pediatr. 116, S98102.

Schwertz, R., Rother, U., Anders, D., Gretz, N., Schärer, K., Kirschfink, M., 2001. Complement analysis in children with idiopathic membranoproliferative glomerulonephritis: a long-term follow-up. Pediatr. Allergy Immunol. 12, 166-172.

Trouw, L.A., Roos, A., Daha, M.R., 2001. Autoantibodies to complement components. Mol. Immunol. 38, 199-206.

Trouw, L.A., Seelen, M.A., Daha, M.R., 2003. Complement and renal disease. Mol. Immunol. 40, 125-134.

Walport, M.J., 2001. Complement. First of two parts. N. Engl. J. Med. 344, 1058-1066.

West, C.D., Witte, D.P., McAdams, A.J., 2001. Composition of nephritic factor-generated glomerular deposits in membranoproliferative glomerulonephritis type 2. Am. J. Kidney Dis. 37, 1120-1130. 


\section{Figure legends}

\section{Fig. 1. Identification of anti-factor $B$ autoantibodies.}

(a) Binding of IgG autoantibodies from the patient's plasma and from plasma samples of C3NeF-positive patients to the immobilized complement proteins factor B (CFB), C3, C3b, factor I (CFI) and factor H (CFH), or to BSA, was analyzed by ELISA. NHP: normal human plasma, used as control. Data points show means of duplicate determinations and are representative of three experiments. (b) Serial dilutions of the patient's plasma showed dosedependent binding to immobilized factor B in ELISA. (c) Removal of the IgG fraction from the patient's plasma by incubation with Protein G beads resulted in a loss of autoantibody binding to factor B. (d) Dose-dependent binding of the IgG fraction, isolated from the patient's plasma, to factor B (auto-CFB IgG). As a control, binding of IgG isolated from normal human plasma to factor B is shown. (e) Immune complexes were captured using a monoclonal antibody against factor B, and detected with anti-human IgG. Data shown are representative of three experiments with similar results.

\section{Fig. 2. Binding specificity of the autoantibody.}

Factor B-specificity was verified in competition experiments. (a) Autoantibody binding from the patient's plasma to immobilized factor B was measured by ELISA in the presence of the indicated concentrations of soluble factor B and C3. (b) A polyclonal goat anti-factor B antibody dose-dependently inhibited autoantibody binding to immobilized factor B, whereas a goat anti-factor $\mathrm{H}$ antibody had no effect. Data are representative of three independent experiments.

\section{Fig. 3. The autoantibodies bind to the Bb part of factor $B$.}

Binding of IgG antibodies to immobilized factor B from the patient's plasma was measured after the factor B-coated wells had been preincubated with increasing concentrations of anti- 
$\mathrm{Ba}$ and anti-Bb monoclonal antibodies. Data are representative of three independent experiments.

Fig. 4. Anti-factor B autoantibodies bind to and stabilize the C3bBb convertase.

(a) Solid-phase alternative pathway C3 convertase was built up on microtiter plates by incubating factor B (B) with factor D (D) on immobilized C3b in the presence of properdin (P) as described by Hourcade et al. (2002). The assembly of the convertase was shown by the presence of the $\mathrm{Bb}$ fragment measured by a polyclonal anti-factor B antibody in ELISA (left panel). This convertase was functionally active and could cleave C3. $10 \mu \mathrm{g} / \mathrm{ml} \mathrm{C3}$ was added for $60 \mathrm{~min}$, and the amount of the generated C3a was measured by ELISA (right panel). (b) IgG isolated from the anti-factor B autoantibody positive patient (auto-CFB IgG) and from C3NeF-containing plasma (C3NeF IgG) were tested for C3 convertase binding in comparison to control IgG. Convertase decay was followed after addition of $500 \mu \mathrm{g} / \mathrm{ml}$ purified IgG (c) in the absence (spontaneous decay) or (d) in the presence of $1 \mu \mathrm{g} / \mathrm{ml}$ factor $\mathrm{H}(\mathrm{CFH})$ (factor $\mathrm{H}$ mediated decay). Data are representative of three independent experiments.

Fig. 5. Anti-factor B autoantibodies inhibit complement-mediated lysis of rabbit erythrocytes.

(a) Rabbit red blood cells were incubated with $10 \%$ human plasma without (-) or with the addition of $1 \mathrm{mg} / \mathrm{ml}$ purified IgG fractions (in PBS) for $30 \mathrm{~min}$ at $37^{\circ} \mathrm{C}$, and complementmediated lysis was measured as hemoglobin release at $414 \mathrm{~nm}$. In a control tube, the same volume of PBS was added as a buffer control (labeled 'PBS'). Polyclonal antibodies against factor B and C3 were also used as controls. (b) The lysis inhibitory effect of anti-factor B autoantibodies and the lysis enhancing effect of C3NeF were dose-dependent. C3a (c) and C5a (d) were measured from the supernatants by ELISA. The effect of C3NeF ( $1 \mathrm{mg} / \mathrm{ml})$ is 
shown as a control. Data represent means of duplicates and are representative of three independent experiments.

Fig. 6. Analysis of the complement activating capacity of autoantibodies and the presence of anti-C1q autoantibodies.

(a) Antigen microarray was used to measure complement activation by autoantibodies in plasma samples. IgG binding and C3 deposition on factor B (circles) and C1q (triangles) from control plasma (white symbols) and from the patient's plasma (black symbols) are shown. Note the logarithmic scale of the $y$-axis. RFU: relative fluorescence units. Data are representative of three experiments. (b) Immobilized purified IgG fractions (20 $\mu \mathrm{g} / \mathrm{ml})$ from the patient with anti-factor B autoantibody and from a healthy individual were analyzed for the presence of C1q by ELISA. (c) IgG binding to immobilized C1q in high-salt (1 M NaCl) buffer was measured from plasma samples to detect anti-C1q autoantibodies. NHP: normal human plasma. Data are representative of two experiments. 
Strobel et al. 2009

\section{Figure 1}

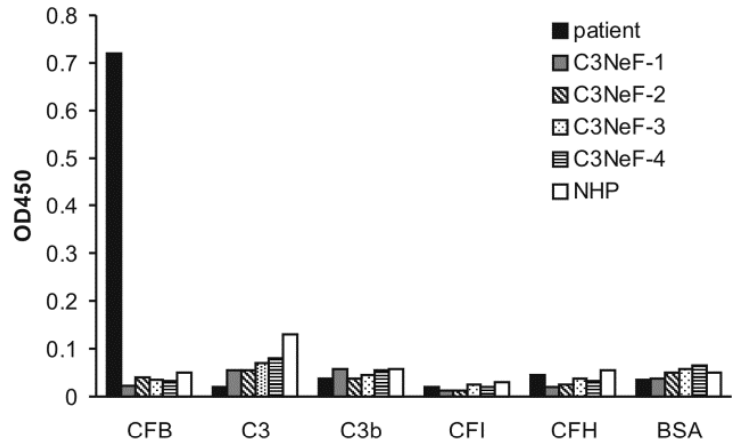

C

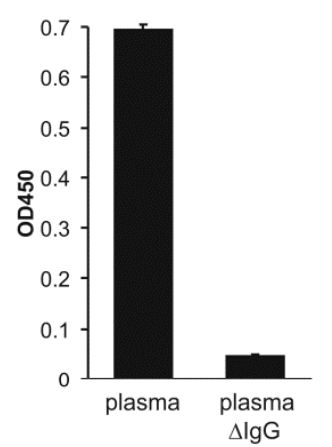

d

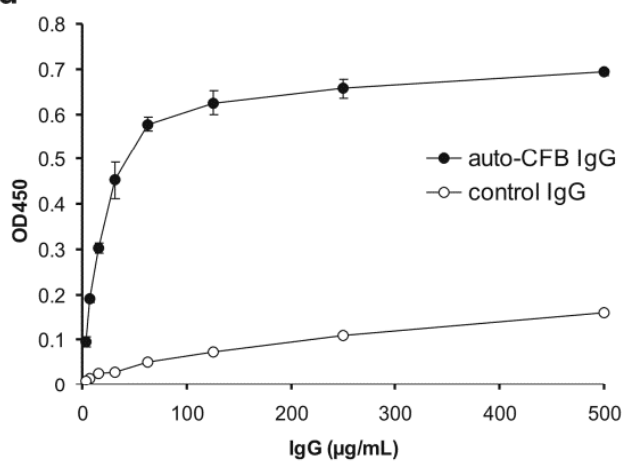

b
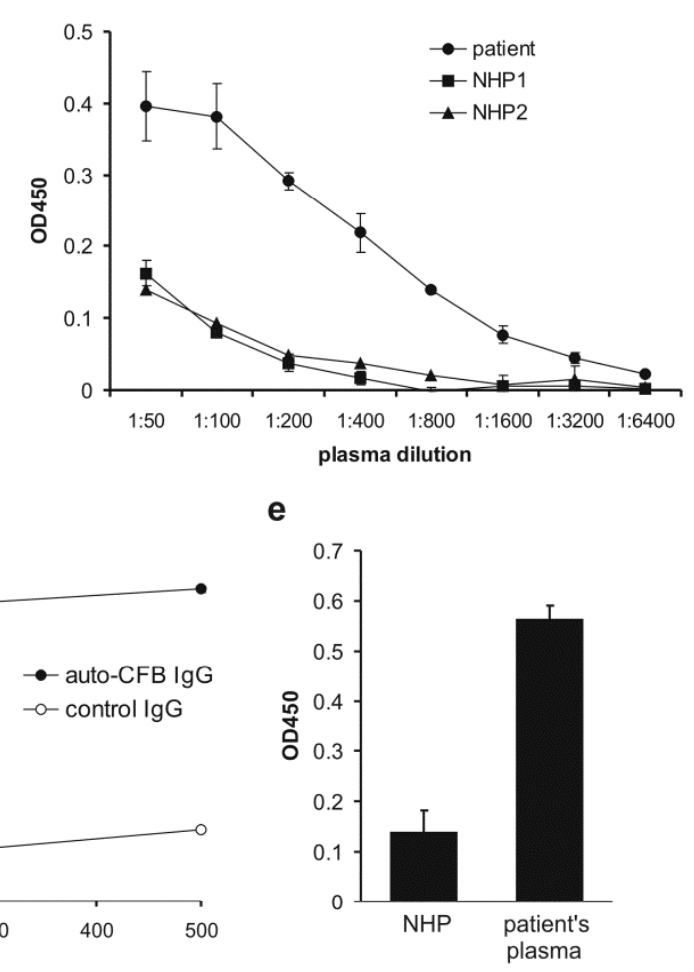
Strobel et al. 2009

\section{Figure 2}
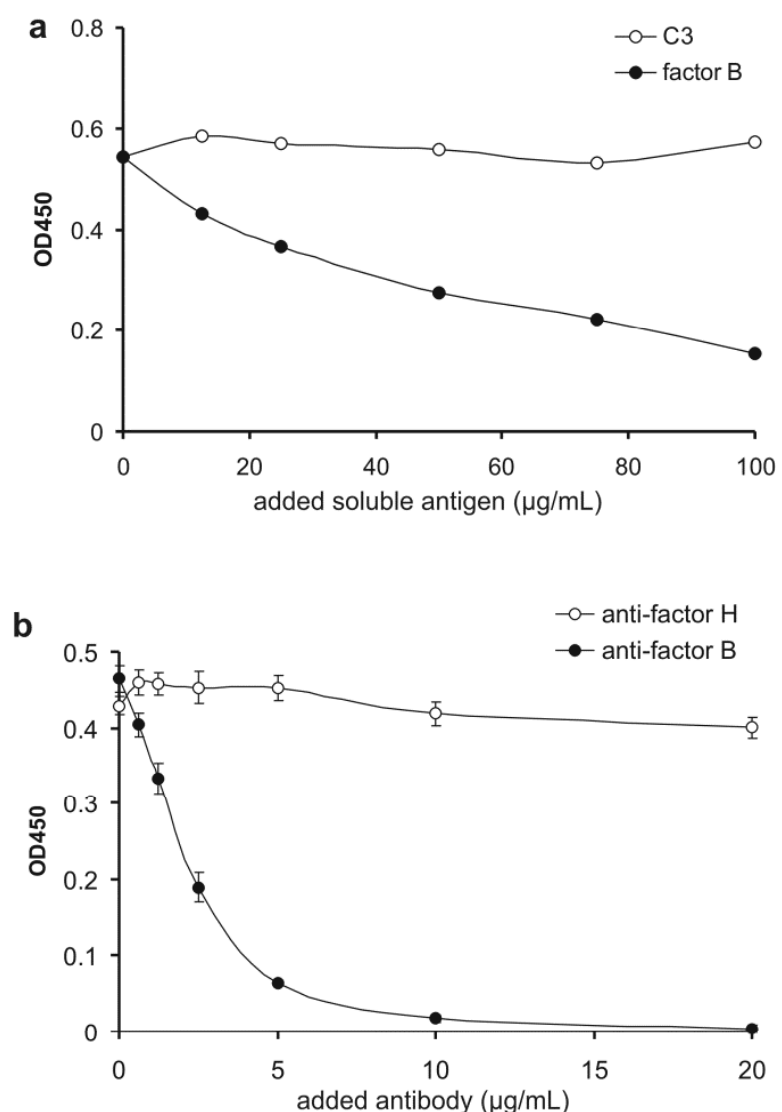

Strobel et al. 2009

Figure 3

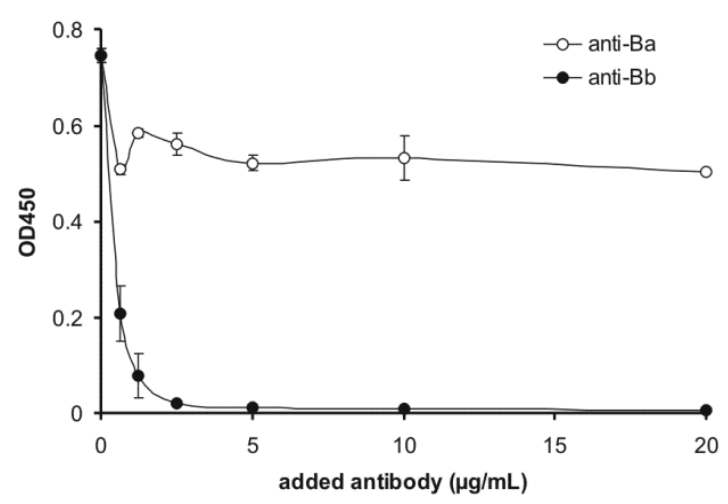


Strobel et al. 2009

Figure 4

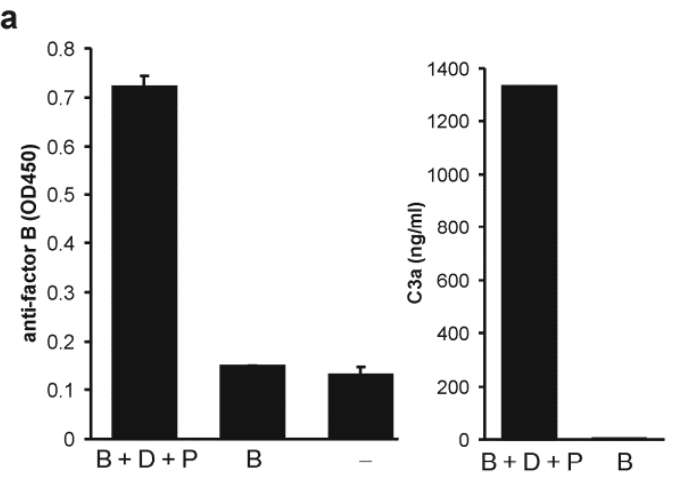

b

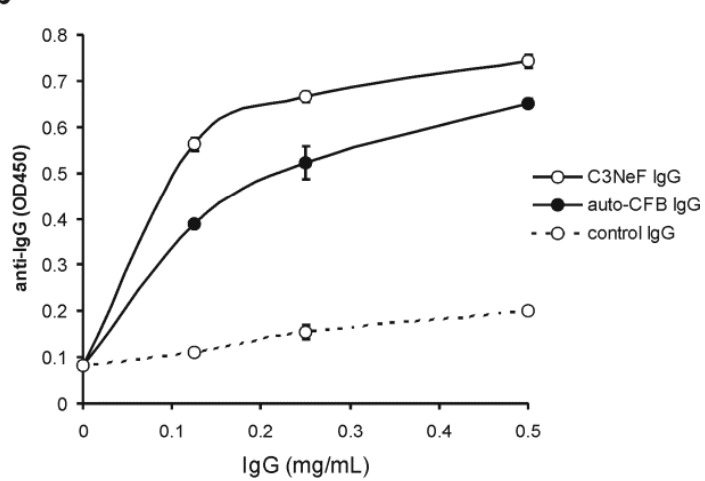

d
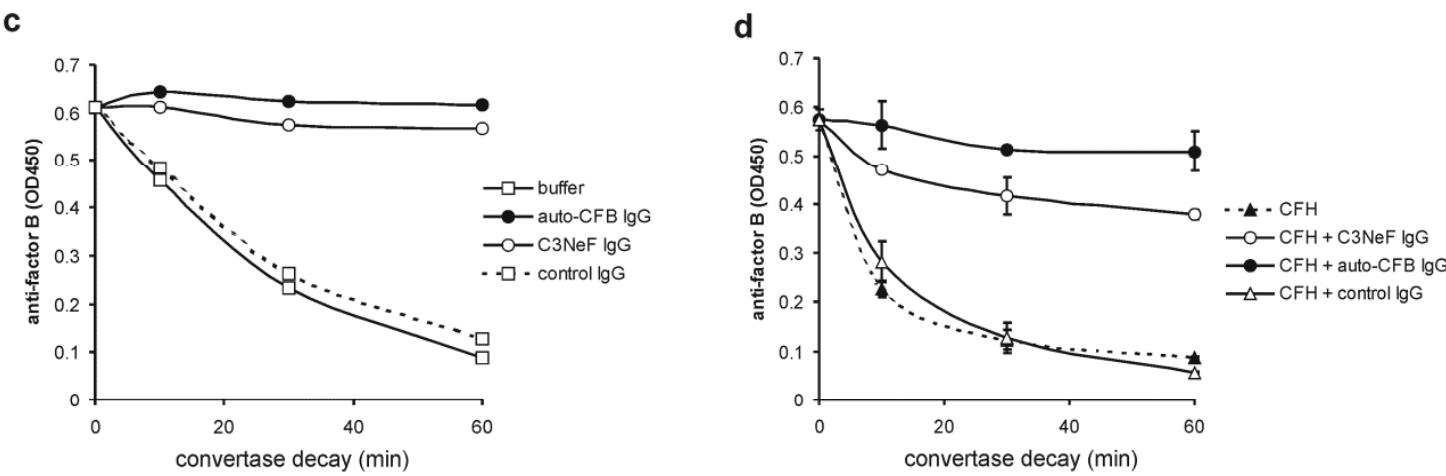
Strobel et al. 2009

Figure 5
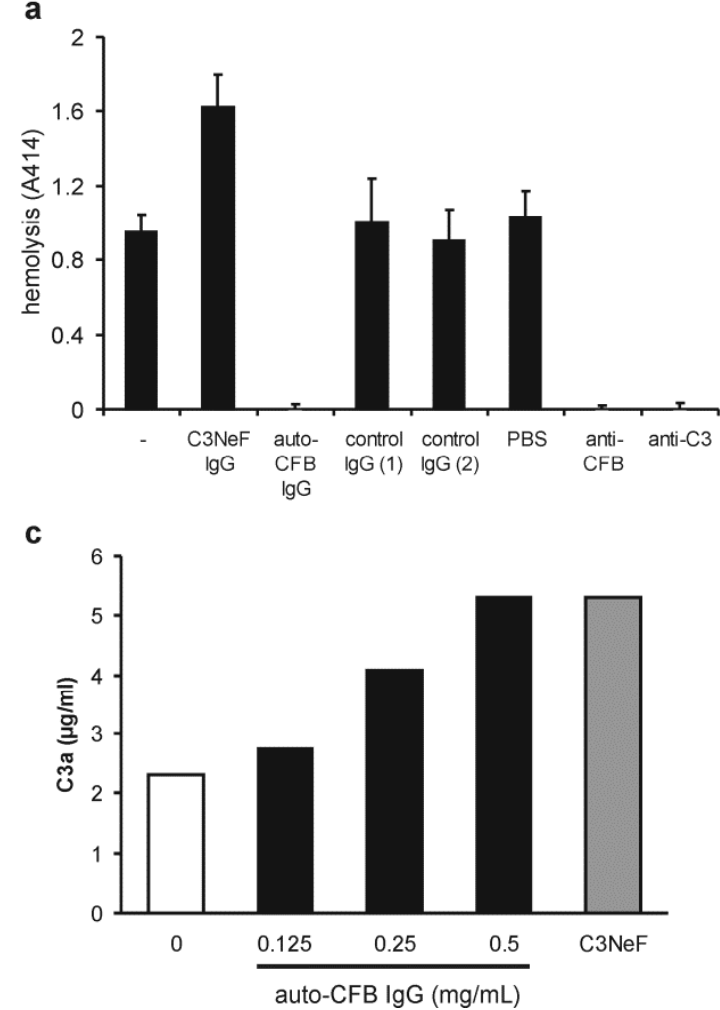

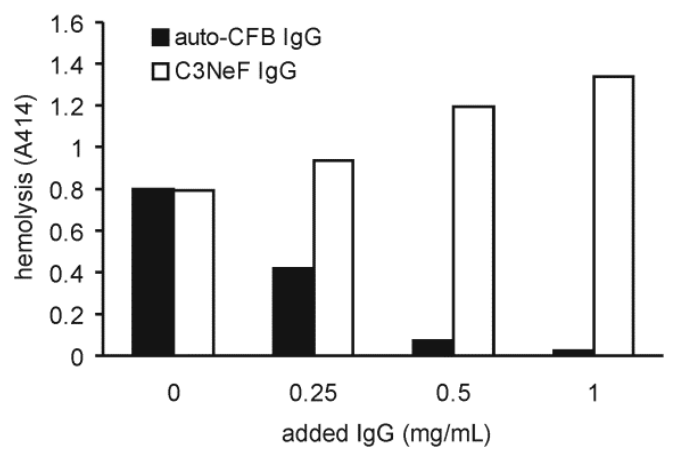

d

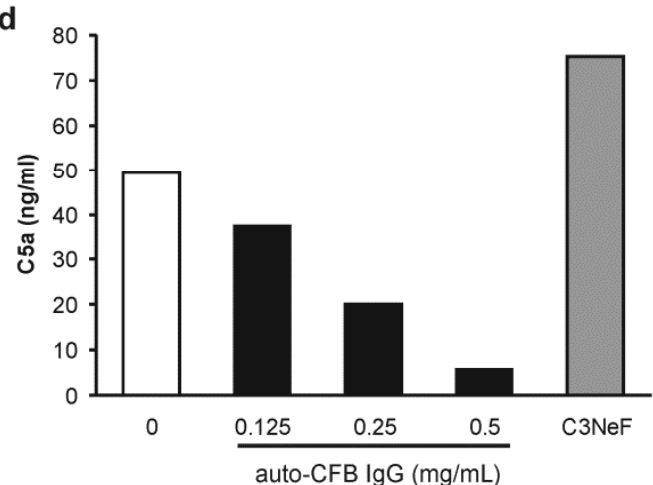


Strobel et al. 2009 Figure 6
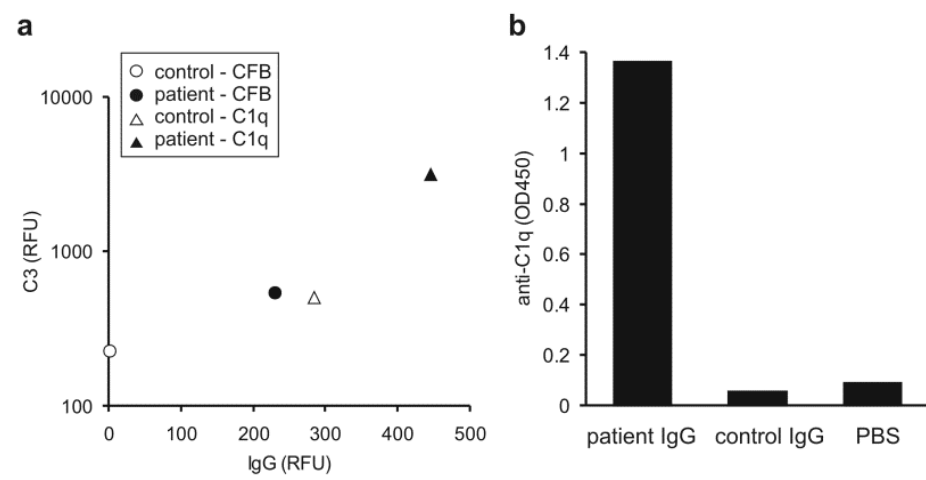

c

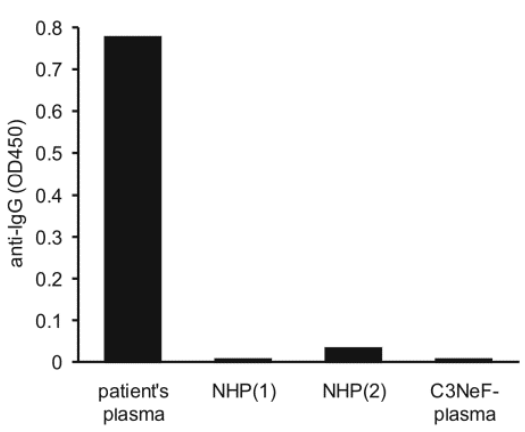

